$\underline{\text { Review Article }}$

\title{
A REVIEW ON PHYSICO-CHEMICAL PARAMETERS OF LIPOSOMAL DOXORUBICIN
}

\author{
DEEPANMOL SINGH ${ }^{1}$, SHILPA PAHWA ${ }^{2}$ \\ ${ }^{1}$ University of Petroleum and Energy Studies, UPES, Bidholi, 248007, Dehradun, India, ${ }^{2}$ Lloyd Institute of Management and Technology \\ (Pharm.), 201306, Greater Noida, India \\ Email: deepanmol.niper@gmail.com
}

Received: 13 Aug 2019, Revised and Accepted: 16 Dec 2019

\begin{abstract}
The development of a generic liposomal doxorubicin product requires the study of critical physicochemical properties of the formulation. Food and Drug Administration (FDA) draft guideline has suggested few parameters to be tested for in vitro bioequivalence study which include liposomal composition, state of encapsulated drug, internal environment, liposomal morphology and number of lamellae, lipid bilayer phase transition liposomal size distribution, grafted Polyethylene Glycol (PEG) at liposomal surface, electric surface potential or charge and in vitro leakage under multiple conditions. Characteristic features of components of liposomal doxorubicin formulation and detail of parameters to be studied have been discussed. This review compile specific, current and historical research outcomes on in vitro analysis of liposomal doxorubicin and highlights the important features that have a critical impact on properties of liposomal doxorubicin formulation. It will provide a better insight to the generic manufacturers and will help them to identify the critical quality attributes during the formulation development phase.
\end{abstract}

Keywords: Bioequivalence, Doxorubicin, Liposome, Critical, Attributes

(C) 2020 The Authors. Published by Innovare Academic Sciences Pvt Ltd. This is an open-access article under the CC BY license (http://creativecommons.org/licenses/by/4.0/) DOI: http://dx.doi.org/10.22159/ijap.2020v12i2.35330. Journal homepage: https://innovareacademics.in/journals/index.php/ijap

\section{INTRODUCTION}

Doxorubicin is a member of the anthracycline class of antineoplastic drugs and is effective in a variety of solid tumours and hematological malignancies [1]. Doxorubicin (Dox) hydrochloride $\left(\mathrm{C}_{27} \mathrm{H}_{29} \mathrm{NO}_{4}\right)$ is a high molecular weight compound $(579.98 \mathrm{gm} / \mathrm{mol})$ and is isolated from Streptomyces peucetius. It was first introduced in the 1970s, and since then it has become one of the most commonly used drugs for the treatment of both hematological and solid tumors [2].

The therapy-limiting toxicity for this drug is cardiomyopathy, which may lead to congestive heart failure and death. Around $2 \%$ of patients who have received Dox experience of this condition. As the usage of Dox is limited to its dose-related cardiotoxicity and myelosuppression, the liposomal form of Dox is beneficial due to its superior efficacy and minimum cardiotoxicity $[3,4]$. Some of the commonly available liposomal based doxorubicin products are DOXIL, CAELYX and MYOCET. DOXIL was one of the first drugs to be approved that is delivered using liposome as a carrier [5]. Maryam et al. (2019) have made attempts to reduce the toxicity of doxorubicin by co-administering it with PEGylated liposomal galbanic acid (PLGba) as it could improve the outcome of the chemotherapy even with a reduced dose of PEGylated liposomal doxorubicin (PLD) [6]. Suchismita et al. (2019) formulated controlled release doxorubicin in the nanocomposite form using graphene grafted with chitosan and polyaniline aiming to reduce the toxicity [7]. Srikanth et al. (2019) have formulated doxorubicin proniosomes to reduce the systemic toxicity of the drug [8]

DOXIL/Caelyx has a longer circulation lifetime which is due to the steric barrier provided by the surface-grafted PEG, which leads to large changes in bio-distribution and particularly increased amounts of drug being delivered to the skin. This has advantages for the treatment of skin localized cancers such as Kaposi's sarcoma but disadvantages in the observation of new dose-limiting toxicities such as hand and foot syndrome [9]

While designing liposomal formulations of Doxorubicin, the following criteria's are kept in mind: (a) Doxorubicin is released from the liposomes following intravenous administration, (b) The distribution of doxorubicin following administration of a liposomal formulation is dependent on the biodistribution characteristics of the liposomes, and (c) The biological activity of liposomal doxorubicin is dependent on when, where, and at what rate the drug is released. So a prerequisite knowledge of the chemical and physical properties and the mechanism (s) of action of this drug, is necessary before formulation [10]. Naozumi et al. (2008) has studied different in vitro parameters of liposomal formulations formed with egg phosphatidylcholine (EPC) as phospholipid and combination of EPC and hydrogenated soya phosphatidylcholine (HSPC) as lipid components and compared them with those of DOXIL [11].

Doxorubicin is an amphipathic molecule possessing a waterinsoluble aglycone (adriamycinone: $\mathrm{C}_{21} \mathrm{H}_{18} \mathrm{O}_{9}$ ) and a water-soluble, basic, reducing amino-sugar moiety (daunosamine: $\mathrm{C}_{6} \mathrm{H}_{13} \quad \mathrm{NO}_{3}$ ). Structure of doxorubicin is given in fig. 1.

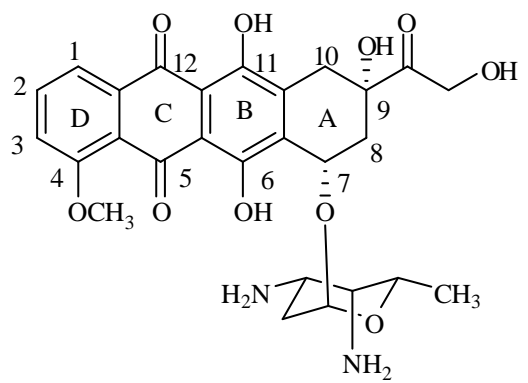

Fig. 1: Structure of doxorubicin

Doxorubicin has anti-mitotic and cytotoxic activity through the formation of complexes with DNA by intercalation between base pairs. It also inhibits topoisomerase II activity by stabilizing the DNA-topoisomerase II complex, preventing the religation portion of the ligation-religation reaction that topoisomerase II catalyzes [1].

Doxorubicin and its major metabolite, doxorubicinol, is $74-76 \%$ bound to plasma protein. The extent to binding is independent of plasma concentration up to $1.1 \mathrm{mcg} / \mathrm{ml}$. Doxorubicin does not cross the blood-brain barrier.

Doxorubicin is capable of undergoing metabolism via 3 metabolic routes: one-electron reduction, two-electron reduction and deglycosylation.

However, approximately half of the dose is eliminated from the body unchanged. Two electron reduction is the primary metabolic pathway and it yields doxorubicinol, secondary alcohol [12]. 


\section{FDA guideline for in vitro testing}

FDA draft guideline gives recommendations regarding the in vitro bioequivalence study of injectable liposomal doxorubicin hydrochloride for product development and quality control of a generic formulation.

FDA's draft guidance for bioequivalence study mentions some of the following parameters which are to be studied. They are: liposomal composition, state of encapsulated drug, internal environment, liposomal morphology and number of lamellae, lipid bilayer phase transition, liposomal size distribution, grafted PEG at the liposomal surface, electric surface potential or charge and in vitro leakage under multiple conditions [13].

\section{Liposomal composition}

In liposomal composition following components are to be evaluated (a) Lipid content, (b) free and encapsulated drug, (c) internal and total sulfate and ammonium concentration, (d) histidine concentration and sucrose concentration [13]. Drug-to-lipid ratio and the percentage of drug encapsulation can be calculated from liposome composition values.

Reports show a strong association of liposomal composition and cytotoxicity and the key factor involved for this is the cellular uptake of the liposomes. PEGylation decreases the intracellular uptake of liposomes and consequently reduces cellular toxicity. Acyl chain length of lipid component influenced the release rate, cytotoxicity increases with short acyl chains as there is an increase in the release of doxorubicin with short acyl chains [14].

DOXIL/Caelyx liposomal formulation contains hydrogenated soya phosphatidylcholine (HSPC), cholesterol (Chol) and PEG-modified phosphatidylethanolamine in 55:40:5 molar ratio whereas Myocet has a different composition as it contains egg phosphatidylcholine (EPC) and Chol in 55:45 molar ratio [15].

Long and fully saturated acyl chains of HSPC confers tight packing of bilayer structure which helps liposomes for better retention due to very low permeability.

Cholesterol eliminates the phase transition of the phospholipids that helps to reduce the permeability of the liposome bilayer to ions and small polar molecules at a temperature range which is critical to the drug loading step during the manufacturing process. Concentration of cholesterol must be sufficient in the bilayer to achieve good drug loading and retention.

Microbubbles coated polyethylene glycol-di-stearoyl phosphatidylethanolamine (MPEG-DSPE) chain confers the "stealth" property to the liposomes. Approximately 5\% of MPEG-DSPE (on the basis of molar ratio) is present in DOXIL. Product with less than 2\% MPEGDSPE showed diminished "stealth" property hence, altered pharmacokinetics [12]. Structures of MPEG-DSPE and HSPC are given in fig. 2-3.

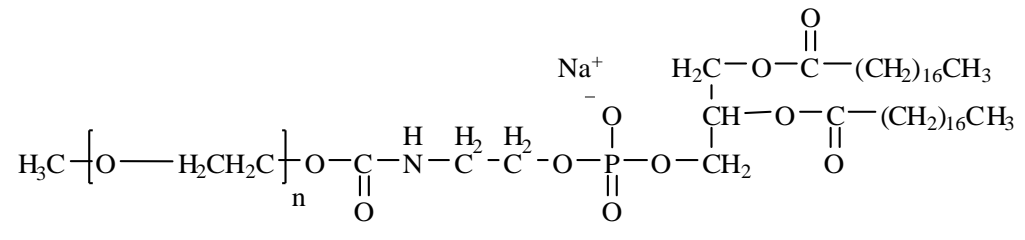

n ca. 45

Fig. 2: Structure of MPEG-DSPE (Microbubbles coated polyethylene glycol-distearoylphosphatidylethanolamine)

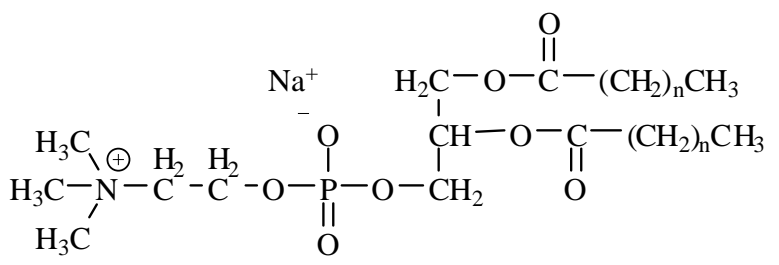

$n, m=14$ or 16

Fig. 3: Structure of HSPC (Hydrogenated soybean phosphatidylcholine)

Mordente et al. (2009) studied and found that the presence of ammonium sulfate at optimal concentration inside the liposomes before the drug loading step is important to achieve high drug loading efficiency [12]. In addition, the sulfate inside of the liposomes complexes with doxorubicin to form a precipitate and is integral to drug retention and drug release.

Cersosimo et al. (1989) found that ion concentrations in a liposome helps to establish a stable and efficient drug encapsulation process. Ammonium ion controls not only the efficiency of the drug loading process, stability of the product and drug release profile of the product. Normal amounts of ammonia for a healthy adult range $0.5 \sim 1 \times 10^{-4} \mathrm{M}$, excess ammonia can potentially lead to severe neurodevelopmental and neurodegenerative complications [16-19].

\section{In vitro leakage testing}

Doxorubicin liposomes are designed to retain doxorubicin during circulation and minimize clearance by the mononuclear phagocyte system and also limit uptake in healthy tissue. Table 1 shows the test conditions recommended by FDA to study the leakage from liposomal doxorubicin.

\section{Factors affecting the release rate}

There are several factors that can affect the release rate. Reported studies showed that only $10 \%$ drug is released in $50 \%$ plasma in normal saline at $37^{\circ} \mathrm{C}$ after $48 \mathrm{~h}$ which could be due to insufficient temperature as the release rate is very low. Release in phosphate buffer solution (at all $\mathrm{pH}$ ) was observed to be higher than the release rate in McLlvaine buffer. Release rate further increased with an increase in dilution. Effect of ultrasound radiation was due to temporary disruption of lipid layer causing rapid release of the drug but with an amplitude-dependent manner. Ultrasound also causes an increase in the particle size.

Shibata et al. (2015) reported that physical stability of bilayer, chemical stability of phospholipids, solubility of drug encapsulated in the liposomes affect the drug release from the liposomes. Increase in the concentration of lysophospholipids and free fatty acids was found to have a clear $\mathrm{pH}$ dependence but ultrasound irradiation does not cause an increase in the concentrations of lysophospholipids or free fatty acids. When the concentration of lysophospholipids and free fatty acids is $12.5 \%$ of total lipids or more permeability of lipid bilayer and hence release rates have been found to increase synergistically at pH 5.5 [20]. 
In a study, conducted by Luo et al. (2016), it was found that Near Infrared radiation (NIR) triggered the release of doxorubicin (Dox) was achieved by including 2 molar \% porphyrin-phospholipid (PoP) from conventional sterically stabilized stealth liposomes [21].

Table 1: Different conditions along with reasons for testing of drug leakage

\begin{tabular}{|c|c|c|c|}
\hline Conditions & Reasons & In vivo organ which it simulates & References \\
\hline $\begin{array}{l}\text { At } 37^{\circ} \mathrm{C} \text { in } 50 \% \text { human plasma for } \\
24 \mathrm{~h} \text {. }\end{array}$ & $\begin{array}{l}\text { Evaluate liposome stability } \\
\text { in blood circulation. }\end{array}$ & Plasma mostly mimics blood conditions. & [13] \\
\hline $\begin{array}{l}\text { At } 37^{\circ} \mathrm{C} \text { with } \mathrm{pH} \text { values } 5.5,6.5 \text {, } \\
\text { and } 7.5 \text { for } 24 \mathrm{~h} \text { in the buffer. }\end{array}$ & $\begin{array}{l}\text { Mimic drug release in } \\
\text { normal tissues, around } \\
\text { cancer cells, or inside cancer } \\
\text { cells. }\end{array}$ & $\begin{array}{l}\text { Normal tissues: pH } 7.3 \\
\text { Cancer tissues: pH } 6.6 \\
\text { Insider cancer cells (endosomes and lysosomes): pH 5-6 } \\
\text { (endosome and lysosomes of cancer cells may be involved in } \\
\text { liposome uptake and induce drug release). }\end{array}$ & {$[13]$} \\
\hline $\begin{array}{l}\text { At a range of temperatures ( } 43 \text {, } \\
\left.47,52 \text { and } 57^{\circ} \mathrm{C}\right) \text { in } \mathrm{pH} 6.5 \text { buffer } \\
\text { for up to } 12 \mathrm{~h} \text { or until complete } \\
\text { release. }\end{array}$ & $\begin{array}{l}\text { Evaluate the lipid bilayer } \\
\text { integrity. }\end{array}$ & $\begin{array}{l}\text { The Tm of lipids is determined by lipid bilayer properties such } \\
\text { as rigidity, stiffness and chemical composition. Differences in } \\
\text { the release as a function of temperature (below or above Tm) } \\
\text { will reflect small differences in lipid properties. }\end{array}$ & {$[13]$} \\
\hline $\begin{array}{l}\text { At } 37^{\circ} \mathrm{C} \text { under low-frequency }(20 \\
\mathrm{kHz}) \text { ultrasound for } 2 \text { h or until } \\
\text { complete release. }\end{array}$ & $\begin{array}{l}\text { Evaluate the state of the } \\
\text { encapsulated drug in the } \\
\text { liposome. }\end{array}$ & $\begin{array}{l}\text { Low-frequency ultrasound ( } 20 \mathrm{kHz} \text { ) disrupts the lipid bilayer } \\
\text { via a transient introduction of pore-like defects and will render } \\
\text { the release of doxorubicin controlled by the dissolution of the } \\
\text { gel inside the liposome. }\end{array}$ & [13] \\
\hline
\end{tabular}

\section{State of doxorubicin}

Doxorubicin is commonly found as a salt form such as sulfate, citrate etc., in the liposomes. Electron microscopy images showed that doxorubicin sulphate containing liposomes were ellipsoidal containing dark stripes. X ray diffraction pattern showed by Lasic et al. (1992) has a single sharp reflection at $27 \AA$ which states that the DOX-sulphate interfibre spacing is $27 \AA$ [22]

In another study on doxorubicin citrate, cryo-Transmission Electron Microscopy (TEM) images showed an interfibre spacing for DOXcitrate to be $30-35 \AA$. NMR studies on ${ }^{13} \mathrm{C}$-citrate revealed that there exists a dynamic interaction between Dox-citrate with the rapid exchange of free and bound citrate.

Studies carried by Xingong et al. (1998) showed that interfibre space difference may be due to the fact that sulphate is a small ion than citrate. DOX-citrate showed different patterns were observed such as hexagonal arrangement, U-shaped arrangement, circular bundles and fibrous bundles with repeating units after $50 \mathrm{~nm}$ [23].

In contrast DOX-sulphate aggregates were noted as only straight bundles in DOX-sulphate.

\section{Internal volume}

The internal environment of a liposome mainly includes volume, $\mathrm{pH}$, sulfate and ammonium concentration helps to maintain the precipitated doxorubicin [14].

Entrapment efficiency is directly related to the internal volume. Entrapment efficiency can be determined by using the following formula:

$$
\mathrm{EE} \%=\left[\frac{\left(\mathrm{C}_{\mathrm{i}}-\mathrm{C}_{\mathrm{f}}\right.}{\mathrm{C}_{\mathrm{i}}}\right] 100
$$

It is an important parameter to measure the morphology of liposomes $[24,25]$. In a study it that relatively low volume of entrapped aqueous space per mole of lipid was observed in the case of both MLV (multilamellar vesicles) and SUV which results in restricted ability to encapsulate large macromolecules. In MLV most of the lipid is participating in the internal lamellae, and there is a restriction of the internal water space due to the close apposition of the concentric adjacent bilayers. In case of SUV, which are single-compartment vesicles, due to large ratio of surface area to encapsulated volume only a small aqueous volume per mole of lipid can be attained [26]. Techniques such as reverse phase evaporation have been developed to prepare liposomes such that a large percentage of aqueous materials can be entrapped in the internal volume [27].

\section{Internal pH}

Liposomes are usually prepared by $\mathrm{pH}$ gradient method either by using citrate buffer or ammonium sulfate buffer. The internal pH was reported to be around 4 in the case of both citrate and ammonium sulfate buffers and was stable for at least $20 \mathrm{~d}$ [28]. Internal $\mathrm{pH}$ has an effect on the stability of the drug. Liposomal system loaded with curcumin was developed with an acidic internal microenvironment $\mathrm{pH}$ has an effect on chemical stability and anticancer activity [29].

\section{Number lamellae and degree of lamellarity}

PEGylated liposomal doxorubincin is an oval-shaped and unilamellar liposome [30]. It is important to study lamellarity as the extent the encapsulation efficiency, drug retention, the efflux rate of liposomally encapsulated material, and the fate of a drug after cellular uptake depends greatly on the lamellarity of liposomes [31, 13]. The degree of lamellarity refers to the number of lipid bilayers in a liposome. A liposome may have a single lipid bilayer, or multiple concentric lipid bilayers. Studies show that DOXIL has approximately 1 to $2 \%$ multilamellarity, the remaining 98 to $99 \%$ of the liposomes have only a single lipid bilayer. When multilamellar vesicles percentage is high, entrapped volume significantly decreases which results in lower drug-to lipid ratio. The net effect is that lower drug is loaded in the liposomes which leads to lower efficiency and higher toxicity. Lower drug release rate has been found in multi-lamellar vesicles as the drug has to pass larger number of lipid bilayer barriers before reaching to the external phase of the liposomes [32].

\section{Particle size distribution}

D50 and Polydispersity index (PDI) given as (D90-D10)/D50 is usually calculated using the population bioequivalence approach (PBE). It is also required to be measured according to the FDA draft guideline along with particle size distribution. To describe the particle size distribution of liposome [33].

The particle size and PDI of nanocarrier systems have a strong influence on the endocytosis-dependent cellular uptake.

Particles smaller than $50 \mathrm{~nm}$ can interact with hepatocytes, while particles larger than $1 \mu \mathrm{m}$ are absorbed by mononuclear phagocytes as emboli [34].

It has been found that nanocarriers with particle size less than 150 $\mathrm{nm}$ are able to cross the fenestrated capillaries in the tumor microenvironment. Tumors have leaky vasculature which allows accumulation of high molecular weight therapeutics in them. This effect is known as the enhanced permeability and retention (EPR) effect. Through this, circulating nanocarriers with size less than around $150 \mathrm{~nm}$ extravasate from the circulation and accumulates within the tumour [35-38]. In some literature, the size below 200 $\mathrm{nm}$ has been mentioned. Reports have shown that by decreasing the diameter size of the liposomes to $50 \mathrm{~nm}$ or below reduced mononuclear phagocyte system (MPS)-mediated clearance in mice 
models with comparable a plasma half-life as of long-circulating (PEGylated) vesicles with a diameter of 100-150 nm [39-40].

\section{Lipid bilayer phase transition}

The gel to the liquid-crystalline phase transition of the lipid bilayer can be determined by differential scanning calorimetery (DSC) and it provides information about bilayer fluidity and uniformity, drug interactions with the lipid bilayer and liposome formation [41]. The fluidity of lipid bilayer depends on the $\mathrm{T}_{\mathrm{C}}$ which is the chain melting transition temperature. Phospholipid exists in different states above and below $\mathrm{T}_{\mathrm{C}}$.

Presence of cholesterol has been reported to enhance the mechanical stability of the membrane. Studies carried by Lorena et al. (2012) showed that low cholesterol contents lead to a phase-segregated system but high cholesterol contents gives a homogeneous bilayer [42].

\section{PEG grafted}

Standard liposome formulations such as Caelyx $\AA$ (DOXIL $\AA$ in the United States) are coated with polyethylene (PEG) which is a synthetic hydrophilic polymer. The bulky PEG head group serves as a barrier preventing interactions with plasma opsonins, hence, it retards recognition by the reticuloendothelial system and slowing elimination of the liposomes from circulation. These PEG-coated liposomes are known as sterically stabilized or STEALTH liposomes (fig. 4) [43].

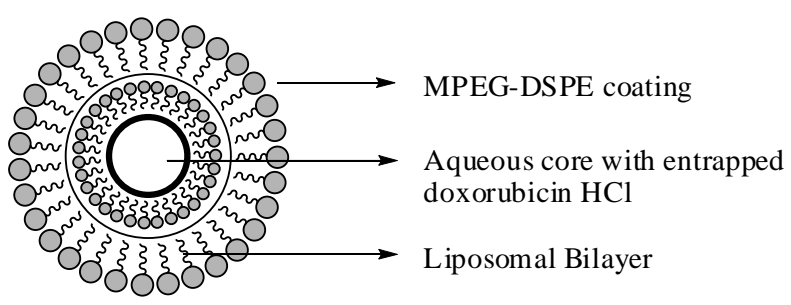

Fig. 4: Structure of stealth lipososme, " ${ }^{\text {MPEG: Microbubbles }}$ coated Poly ethylene glycol, DSPE: Distearoyl phosphatidyl ethanolamine

Greater than $90 \%$ of the drug is encapsulated as STEALTH liposomes [23].

Jianwei et al. (2018) co-encapsulated doxorubicin and verapamil into gold liposomes to overcome the multi-drug resistance as verapamil inhibits multi drug resistance protein [44].

PEG grafts can be present in two different configurations: as a random coil grafted in one end ("mushroom") or as an approximately linear polymer ("brush").

Surface PEG conformation (mushroom or brush conformation) determination was based on the calculated ratio of the Flory dimension $\left(R_{\mathrm{f}}\right)$ to the average distance between adjacent PEG chains $(D)$. PEG Flory dimension and the distance between surface grafted PEG chains were calculated using the given equation.

$$
R_{\mathrm{f}}=a N^{3 / 5}
$$

Where $a$ is PEG monomer size in $\AA$ (previously reported as $3.8 \AA$ [45], $N$ is the degree of polymerization,

$$
\mathrm{D}=\left(\frac{\mathrm{A}}{\mathrm{M}}\right)^{1 / 2}
$$

$A$ is the PEG area per lipid molecule in the bilayer (previously reported as $\left.67 \AA^{2}[46]\right)$ and $M$ is the mole fraction of PEG lipid determined experimentally as described above $R_{\mathrm{f}} / D$ values calculated below 1.0 indicates a mushroom regime, while those above 1.0 indicate brush [47].

\section{Electric surface potential or charge}

Surface charge (zeta potential) on liposomes has a significant effect on the clearance, tissue distribution and cellular uptake.
Cancer cell surfaces are usually charged negatively due to the translocation of negatively charged constituents of the inner layer of the cell membrane (e. g., phosphatidylserine, anionic phospholipids, glycoproteins and proteoglycans), the cationic liposomal doxorubicin (LPs-DOX) will have increased uptake due to better interaction between negatively charged cancer cells and positively charged (LPs-DOX) [48].

A strong linear relationship was noted between zeta potential values and the mole percentage of charged lipids within a liposome [49].

Negatively charged liposomes have been reported to be immunostimulant but zwitterionic ones were not. [50-51].

\section{CONCLUSION}

Parameters for in vitro bioequivalence study for generic product development of liposomal doxorubicin have been described in FDA draft guideline. The review helps to understand the importance of each physicochemical parameter critical for the formulation of a liposomal form of doxorubicin. Role of each component has been described which will be useful in formulation development scientist as well as to an analytical chemist.

\section{FUNDING}

Nil

\section{AUTHORS CONTRIBUTIONS}

All the authors have contributed equally.

\section{CONFLICT OF INTERESTS}

Declared none

\section{REFERENCES}

1. Hortobagyi GN. Anthracyclines in the treatment of cancer: an overview. Drugs 1997;54:1-7.

2. Niu G, Cogburn B, Hughes J. Preparation and characterization of doxorubicin liposomes. Methods Mol Biol 2010;624:211-9.

3. Tacar O, Sriamornsak P, Dass CR. Doxorubicin: an uptake on anticancer molecular action, toxicity and novel drug delivery systems. J Pharm Pharmacol 2013;65:157-70.

4. Noveen K, Arvind G, Sumit S, Prashanth P. Formulation and evaluation of non-pegylated doxorubicin liposomal drug delivery system. Int J Pharm Pharm Sci 2013;5:541-7.

5. http://www.horiba.com/fileadmin/uploads/Scientific/Docum ents/PSA/AN174_app.pdf [Last accessed on 12 Jun 2019]

6. Maryam EN, Bizhan M, Mohamadreza A, Mahdi H, Manouchehr T, Hamid RS, et al. Liposomal formulation of galbanic acid improved therapeutic efficacy of pegylated liposomal doxorubicin in mouse colon carcinoma. Sci Rep 2019;9:9527.

7. Suchismita M, Subrata S, Gouri SR. Formulation and evaluation of graphene grafted chitosan/polyaniline nanocomposites for controlled release of anticancer drug doxorubicin. Int J Appl Pharm 2019:11:138-43.

8. Srikanth, Anand KY, Mallikarjuna SC. Design and optimization of doxorubicin $\mathrm{HCl}$ proniosomes by design of experiment. Int J Pharm Pharm Sci 2019;11:90-5.

9. Waterhouse DN, Tardi PG, Mayer LD, Bally MB. A comparison of liposomal formulations of doxorubicin with drug administered in free form: changing toxicity profiles. Drug Saf 2001;24:903-20.

10. Bouma J, Beijnen JH, Bult A, Underberg WJ. Anthracycline antitumour agents: a review of physicochemical, analytical and stability properties. Pharm Weekbl Sci Ed 1998;8:109-33.

11. Naozumi O, Hiromasa T, Yousuke I, Tahara K, Hirofumi T. Characterization of a doxorubicin liposome formulation by a novel in vitro release test methodology using column-switching high-performance liquid chromatography. Chem Pharm Bull 2014;62:538-44.

12. Mordente A, Meucci E, Silvestrini A, Martorana GE, Giardina B. New development in anthracycline induced cardiotoxicity. Curr Med Chem 2009;16:1656-72.

13. https://www.accessdata.fda.gov/drugsatfda_docs/psg/Dox orubicin\% 20Hydro chloride_draft_Injection\%20injec\% 
20lipo_RLD\%2050718_RC09-18pdf [Last accessed on 28 May 19].

14. Sakai Kato K, Nanjo K, Kawanishi T, Okuda H, Goda Y. Effects of lipid composition on the properties of doxorubicin-loaded liposomes. Ther Delivery 2015;6:785-94.

15. https://www.liposomes.ca/publications/268\%20Abraham $\% 2$ 0et\%20al\%202005pdf [Last accessed on 12 Jun 2019].

16. Cersosimo E, Williams $P$, Geer $R$, Lairmore $T$, Ghishan $F$, Abumrad NN. Importance of ammonium ions in regulating hepatic glutamine synthesis during fasting. Am J Physiol 1989;257:514-9.

17. Summar ML, Koelker S, Freedenberg D, Le Mons C, Haberle J, Lee HS, et al. The incidence of urea cycle disorders. Mol Genet Metab 2013;110:179-80.

18. Ayyub OB, Behrens AM, Heligman BT, Natoli ME, Ayoub JJ, Cunningham G, et al. Simple and inexpensive quantification of ammonia in whole blood. Mol Genet Metab 2015;115:95-100.

19. Bosoi CR, Rose CF. Identifying the direct effects of ammonia on the brain. Metab Brain Dis 2009;24:95-102.

20. Shibata H, Izutsu K, Yomota C, Okuda H, Goda Y. Investigation of factors affecting in vitro doxorubicin release from PEGylated liposomal doxorubicin for the development of in vitro release testing conditions. Drug Dev Ind Pharm 2015;41:1376-86.

21. Luo D, Carter KA, Razi A, Geng J, Shao S, Giraldo D, et al. Doxorubicin encapsulated in stealth liposomes conferred with light-triggered drug release. Biomaterials 2016;75:193-202.

22. Lasic DD, Frederik PM, Stuart MCA, Barenholz Y, McCintosh TJ. Gelation of liposome interior. A novel method for drug encapsulation. FEBS Lett 1992;312:255-8.

23. Xingong L, Hirsh DJ, Cabral Lilly D, Zirkel A, Gruner SM, Janoff $\mathrm{AS}$, et al. Doxorubicin physical state in solution and inside liposomes loaded via a pH gradient. Biochim Biophys Acta 1998;1415:23-40

24. Cagdas M, Sezer AD, Bucak S. Liposomes as potential drug carrier systems for drug delivery. Appl Nanotechnol Drug Delivery 2014;1-50. Doi:10.5772/58459

25. Bahareh S, Mohamed IN, Shaharuddin M, Rosnani H, Afendi D, Hamid AJ. Development and characterization of liposomal doxorubicin hydrochloride with palm oil. BioMed Res Int 2014. http://dx.doi.org/10.1155/2014/765426

26. AD Bangham. Membrane models with phospholipids. Prog Biophys Mol Biol 1968;18:29-95.

27. Szoka F, Papahadjopoulos D. Procedure for preparation of liposomes with large internal aqueous space and high capture by reverse-phase evaporation. Proc Natl Acad Sci USA; 1978. p. 4194.

28. Chemin C, Pean JM, Bourgaux C, Pabst G, Wuthrich P, Couvreur $\mathrm{P}$, et al. Supramolecular organization of S12363-liposomes prepared with two different remote loading processes. Biochim Biophys Acta Biomembr 2009;1788:926-35.

29. Shao XR, Wei XQ, Zhang S, Fu N, Lin YF, Cai XX, et al. Effects of micro-environmental ph of liposome on chemical stability of loaded drug. Nanoscale Res Lett 2017;12:504.

30. Roces CB, Kastner E, Stone P, Lowry D, Perrie Y. Rapid quantification and validation of lipid concentrations within liposomes. Pharmaceutics 2016;8:29.

31. Frohlich M, Brecht V, Peschka Suss R. Parameters influencing the determination of liposome lamellarity by ${ }^{31} \mathrm{P}-\mathrm{NMR}$. Chem Phys Lipids 2001;109:103-12.

32. Citizen petition FDA-2009-P-0216 Ortho-Biotech Products, L. P; 2009. Available from: www.regulations.gov/search/ Regs/home.html \#documentDetail?R=09000064809878d9 [Last accessed on 12 Jun 2019].
33. Jiang $\mathrm{W}$, Lionberger $\mathrm{R}$, $\mathrm{Yu}$ L. In vitro and in vivo characterizations of PEGylated liposomal doxorubicin. Bioanalysis 2011;3:333-44.

34. Hui Y, Hao L, Jun Z. Preparation of prolonged-circulating galangin-loaded liposomes and evaluation of antitumor efficacy in vitro and pharmacokinetics in vivo. J Nanomater 2019. https://doi.org/10.1155/2019/7236895

35. Mozafari MR, Pardakhty A, Azarmi S, Jazayeri JA, Nokhodchi A, Omri A. Role of nanocarrier systems in cancer nanotherapy. J Liposome Res 2009;19:310-21.

36. Aw-Yong PY, Gan PH, Sasmita AO, Mak ST, Ling APK. Nanoparticles as carriers of phytochemicals: recent applications against lung cancer. Int J Res Biomed Biotechnol 2018;7:1-11.

37. Greish $\mathrm{K}$, Fang J, Inutsuka $\mathrm{T}$, Nagamitsu A, Maeda $\mathrm{H}$. Macromolecular therapeutics. Clin Pharmacokinet 2003;42:1089105.

38. Thi Lan Nguyen, Thi Hiep Nguyen, Dai Hai Nguyen. Development and in vitro evaluation of liposomes using soy lecithin to encapsulate paclitaxel. Int J Biomater 2017. https://doi.org/10.1155/2017/8234712

39. Gabizon AA, Barenholz Y, Bialer M. Prolongation of the circulation time of doxorubicin encapsulated in liposomes containing a polyethylene glycol-derivatized phospholipid: pharmacokinetic studies in rodents and dogs. Pharm Res 1993;10:703-8.

40. Proffitt RT, Williams LE, Presant CA, Tin GW, Uliana JA, Gamble $\mathrm{RC}$, et al. Tumor-imaging potential of liposomes loaded with In111-NTA: biodistribution in mice. J Nucl Med 1983;24:45-51.

41. Demetzos C. Differential scanning calorimetry (DSC): a tool to study the thermal behavior of lipid bilayers and liposomal stability. J Liposome Res 2008;18:159-73.

42. Lorena R, Marina IG, Fausto S. Influence of cholesterol on the phase transition of lipid bilayers: a temperature-controlled force spectroscopy study. Langmuir 2012;28:12851-60.

43. https://www.ema.europa.eu/en/documents/comments/overv iew-comments-receivedpegylatedliposomaldoxorubicinhydrochloride-concentrate-solution-2-mg/ml-product-specificbioequivalenceguidance-ema/chmp/800775/2017_en.pdf [Last accessed on 12 Jun 2019]

44. Jianwei J, Shaojuan L, Chunlei W, Hongyan Zhang. Overcoming multidrug resistance by on-demand intracellular release of doxorubicin and verapamil. J Nanomaterials 2018. https://doi.org/10.1155/2018/3568190

45. Zeng $\mathrm{L}, \mathrm{Wu} \mathrm{X}$. Modeling the sustained release of lipophilic drugs from liposomes. Appl Phys Lett 2010;97. https://doi.org/10.1063/1.3479924

46. Alwarawrah M, Dai J, Huang J. A molecular view of the cholesterol condensing effect in DOPC lipid bilayers. J Phys Chem B 2010;114:7516-23.

47. Labouta HI, Gomez Garcia MJ, Sarsons CD, Nguyen T, Kennard J, Ngo W, et al. Surface-grafted polyethylene glycol conformation impacts the transport of PEG-functionalized liposomes through a tumour extracellular matrix model. RSC Adv 2018;8:7697-708.

48. Honary S, Zahir F. Effect of zeta potential on the properties of nano-drug delivery systems-a review (Part 1). Trop J Pharm Res 2013;12:255-64.

49. Smith MC, Crist RM, Clogston JD, Mc Neil SE. Zeta potential: a case study of cationic, anionic, and neutral liposomes. Anal Bioanal Chem 2017;409:5779-87.

50. Byrne JD, Betancourt T, Brannon Peppas L. Active targeting schemes for nanoparticle systems in cancer therapeutics. Adv Drug Delivery Rev 2008;60:1615-26.

51. Csaba N, Garcia Fuentes M, Alonso MJ. Nanoparticles for nasal vaccination. Adv Drug Delivery Rev 2009;61:140-57. 